graduate courses and the relative frequency of their occurrence. In the present paper a similar study is made of the calculus used by the practicing engineers, the more recent volumes of leading technical journals furnishing the data used. He discusses also the emphasis placed on calculus and the attitude of the engineers of different nationalities toward it.

12. Mr. Smail considers a hypercomplex number system in two units $e_{1}, e_{2}$, where $e_{1}^{2}=e_{1}, e_{1} e_{2}=e_{2} e_{1}=0, e_{2}^{2}=e_{2}$. He considers the elementary conceptions and the functionality of the simpler algebraic expressions. Differentiation is defined and its limitations pointed out; integration is defined and the integral of a hypercomplex variable expressed in terms of ordinary curvilinear integrals. He finds also the conditions that make the integral independent of the path of integration.

H. C. Moneno, Secretary of the Section.

\title{
INVARIANT CONDITIONS THAT A $p$-ARY FORM MAY HAVE MULTIPLE LINEAR FACTORS.
}

BY PROFESSOR O. E. GLENN.

(Read before the American Mathematical Society, October 29, 1910.)

\section{§1. Introduction.}

In the case of a ternary quadratic form the vanishing of the ordinary discriminant is the necessary and sufficient condition that the form be factorable into linear factors, distinct term for term. In the case of a cubic ternary form we can obtain in various ways a set of three independent rational, integral, and homogeneous functions of the coefficients, whose simultaneous vanishing furnishes the necessary and sufficient conditions that the form be factorable into distinct linear factors. The general theorem underlying these facts has been proved by Junker,* and is that the necessary and sufficient conditions that a $p$-ary form of order $m$ be a product of $m$ distinct linear factors consist in the simultaneous vanishing of a set of $\left(\begin{array}{c}m+p-1 \\ m\end{array}\right)-m(p-1)-1$ independent seminvariant $\uparrow$ relations among the form's coefficients. 45 .

*Junker, "Die symmetrischen Funktionen u. s. w.," Math. Annalen, vol. 64.

$\nmid$ Junker, "Ueber die Diff.-Gleichungen der Invarianten," loc. cit., vol. 
Several papers* have been published on the problem of determining such sets of conditions. Brill $\uparrow$ and Gordan $\ddagger$ have each treated the problem by the symbolical methods, obtaining in each case a redundant set of conditions furnished by the identical vanishing of a covariant. Junker confined his attention to the problem of finding in terms of the coefficients the $\left(\begin{array}{c}m+p-1 \\ m\end{array}\right)-1$ identical relations which exist among the elementary symmetric functions of the $m$ groups of $p$ homogeneous variables of the form. His sets of relations also do not contain the minimum number. There exist $m(p-1)$ syzygies among them.

Not much attention seems to have been given however to the problem of determining a minimum set of conditions that a $p$-ary form $f_{p m}(p>2)$ may possess multiple linear factors. It is the purpose of this note to show how a certain factor theorem for $f_{3 m}$, which we give in the first part of $\S 2$, may be made the basis of various methods of determining such sets. In $\$ 2$ we determine conditions in order that a $p$-ary $m$-ic $f_{p m}$ should be a perfect $m$ th power. In $\S 3$ sets of conditions that $f_{3 m}$ should involve a repeated factor are developed. Proof that the sets of conditions determined in the various cases really contain the minimum number is given in $\S 4$.

$\S 2$. Conditions that a p-ary Form may be an mth Power.

Let

(1) $f_{3 m}=x_{2}^{m} \phi_{0 x_{1} \mid x_{2}}+x_{2}^{m-1} \phi_{1 x_{1} / x_{2}} x_{3}+x_{2}^{m-2} \phi_{2 x_{1} / x_{2}} x_{3}^{2}+\cdots+\phi_{m} x_{1} \mid x_{2} x_{3}^{m}$, where

$$
\begin{aligned}
x_{2}^{m-r} \phi_{r x_{1} / x_{2}} \equiv a_{m-r 0} x_{1}^{m-r} & +a_{m-r-11} x_{1}^{m-r-1} x_{2} \\
& +a_{m-r-22} x_{1}^{m-r-2} x_{2}^{2}+\cdots+a_{0 m-r} x_{2}^{m-r},
\end{aligned}
$$

be a ternary form equal to the product of $m$ linear factors. Then the $\left(x_{1}, x_{2}\right)$ terms of those factors must be furnished by the linear factors of the binary form $x_{2}^{m} \phi_{0 x_{1} / x_{2}}$. Let the factors of the latter be assumed to be known and let them be

$$
x_{1}+r_{i} x_{2} \quad(i=1,2, \cdots, t),
$$

* Brioschi, "Sulla condizioni per la decomposizione di una forma cubica ternaria, etc." Annali di Matematica, ser. 12, vol. 7.

† Brill, "Ueber die Zerfällung der Ternärformen in lineare Factoren," Jahresbericht der Deutschen Math.-Vereinigung, 1896, and Math. Annalen, vol. 50.

$\ddagger$ Gordan, " Das Zerfallen der Kurven in gerade Linien," loc. cit., vol. 45. 
where $x_{1}+r_{i} x_{2}$ is of multiplicity $\alpha_{i}$. We have proved in another paper * the following

Theorem : The ternary form $f_{3 m}$ can be factored into factors of the respective orders $\alpha_{1}, \alpha_{2}, \ldots, \alpha_{t}$, which are rational and integral in the coefficients of the form $f_{3 m}$ itself on the one hand, and in the quantities $r_{i}$ on the other, linear in the coefficients; according to the formula

$$
\begin{aligned}
& f_{3 m}=\prod_{i=1}^{t} {\left[\frac{\partial^{\alpha_{i}} \phi_{0-r_{i}}}{\partial r_{i}^{\alpha_{i}}} x^{\alpha_{i}}-\alpha_{i} \frac{\partial^{\alpha_{i}-1} \phi_{1-r_{i}}}{\partial r_{i}^{a_{i}-1}} x^{\alpha_{i}-1} y_{i}+\alpha_{i}\left(\alpha_{i}-1\right)\right.} \\
& \times\left.\frac{\partial^{\alpha_{i}-2} \phi_{2-r_{i}}}{\partial r_{i}^{\alpha_{i}-2}} x^{\alpha_{i}-2} y_{i}^{2}-\cdots+(-1)^{\alpha_{i}} \underline{\alpha_{i}} \phi_{a_{i}-r_{i}} y_{i}^{\alpha_{i}}\right] \\
& x_{1}+r_{i} x_{2} / x_{3}=x / y_{i} \quad(i=1,2, \cdots, t) .
\end{aligned}
$$

If the Hessian covariant of one of these factors of $f_{3 m}$, considered as a binary form in $(x, y)$, vanishes identically, $f_{3 m}$ has a linear factor of multiplicity $\alpha_{i}$, and conversely. Thus if $m=2$,

$$
f_{32}=a x_{1}^{2}+2 h x_{1} x_{2}+b x_{2}^{2}+2 g x_{1} x_{3}+2 f x_{2} x_{3}+c x_{3}^{2},
$$

we get in this manner necessary and sufficient conditions that the general ternary quadratic be a perfect square. These are $t$

$$
\begin{gathered}
C_{1}=a f^{2}-2 f g h+b g^{2}=0, \\
C_{2}=a b-h^{2}=0, \quad C_{3}=a c-g^{2}=0 .
\end{gathered}
$$

They are independent, that is, as we shall show in general in $\S 4$, they form a minimum set of conditions that a general form $f_{32}$ be a perfect square.

We proceed now to generalize the results in the last paragraph, deriving necessary and sufficient conditions that the factorable $p$-ary form $f_{p m}$ be a perfect $m$ th power.

A notation can be adopted for $f_{p m}$ which exhibits the form as a sum of $p-2$ ternary forms plus a residue $\Psi_{p m}$ which is not in the shape of a ternary form. We use for the ternary summands a notation like (1), e. g.,

* Bulletin, vol. 17, No. 2, p. 63 .

†Cf. Bromwich, Quadratic Forms, p. 9. 


$$
\begin{aligned}
& f_{p m}=x_{2}^{m} \phi_{0 x_{1} / x_{2}} \\
& +x_{2}^{m-1} x_{3} \phi_{1 x_{1} / x_{2}}^{(1)}+x_{2}^{m-2} x_{3}^{2} \phi_{2 x x_{2}}^{(1)}+\cdots+x_{3}^{m} \phi_{m x_{1} \mid x_{2}}^{(1)} \\
& +x_{2}^{m-1} x_{4} \phi_{1 x_{1} / x_{2}}^{(2)}+x_{2}^{m-2} x_{4}^{2} \phi_{2 x_{1} / x_{2}}^{(2)}+\cdots+x_{4}^{m} \phi_{m x_{1} \mid x_{2}}^{(2)} \\
& +x_{2}^{m-1} x_{p} \phi_{1 x_{1} / x_{2}}^{(p-2)}+x_{2}^{m-2} x_{p}^{2} \phi_{2 x_{1} / x_{2}}^{(p-2)}+\cdots+x_{p}^{m} \phi_{m x_{1} / x_{2}}^{(p-2)} \\
& +\Psi_{p m} \text {. }
\end{aligned}
$$

When assumed to be linearly factorable, this equals

$$
f_{p m}=\prod_{i=1}^{m}\left(r_{1 i} x_{1}+r_{2 i} x_{2}+r_{3 i} x_{3}+\cdots+r_{p i} x_{p}\right),
$$

and if we take

$$
\begin{aligned}
& a_{k_{1} k_{2} \ldots k_{h} \ldots k_{p}}=\sum r_{11} r_{12} \cdots r_{1 k_{1}} r_{2 k_{1}+1} r_{2 k_{1}+2} \\
& \cdots r_{2 k_{1}+k_{2}} \cdots r_{h k_{1}+k_{2}+\cdots+k_{h}} \cdots r_{p k_{1}+k_{2}+\ldots+k_{p}} \text {, }
\end{aligned}
$$

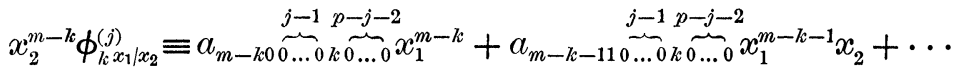

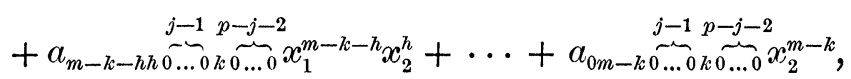

showing the notation for the coefficients of the form $f_{p i n}$. We note that $\phi_{0 x_{1} \mid x_{2}}^{(j)} \equiv \phi_{0 x_{1} / x_{2}}$; and we take, without loss of generality, $a_{m 00 \ldots}=1$.

Assume that the roots of $\phi_{0,-r}$ are all equal. Then $x_{2} \phi_{0 x_{1} \mid x_{2}}$ is the $m$ th power of a linear factor $x_{1}+r_{1} x_{2}\left(r_{21}=r_{1}\right)$. As is well known, a* necessary and sufficient condition for this is that the Hessian $H \phi_{0}$ of $x_{2}^{m} \phi_{0 x_{1} / x_{2}}$ should vanish identically. By (2), when $H \phi_{0} \equiv 0$, the typical ternary form in (3), $X^{(j)}$, say,

$$
X^{(j)}=x_{2}^{m} \phi_{0 x_{1} / x_{2}}+x_{2}^{m-1} x_{j+2} \phi_{1 x_{1} / x_{2}}^{(j)}
$$

is expressible in the form

$$
+x_{2}^{m-2} x_{j+2}^{2} \phi_{2 x_{1} / x_{2}}^{(j)}+\cdots+x_{j+2}^{m} \phi_{m x_{1} / x_{2}}^{(j)},
$$

$$
\begin{aligned}
X^{(j)} & \equiv \frac{\partial^{m} \phi_{0-r_{1}}^{(j)}}{\partial r_{1}^{m}} x^{m}-m \frac{\partial^{m-1} \phi_{1}^{(j)}-r_{1}}{\partial r_{1}^{m-1}} x^{m-1} y \\
& +m(m-1) \frac{\partial^{m-2} \phi_{2}^{(j)}-r_{1}}{\partial r_{1}^{m-2}} x^{m-2} y^{2}-\cdots+(-1)^{m}\left\lfloor m \phi_{m-r_{1}}^{(j)} y^{m},\right.
\end{aligned}
$$

* Other criteria may be had ; for instance, a necessary and sufficient condition that a binary $n$-ic $f$ be an $n$th power is that the matrix of another binary $n$-ic $\phi$ with the bilinear invariant of $f$ and $\phi$ should have an invariant factor different from unity. 
where $x / y=\left(x_{1}+r_{1} x_{2}\right) / x_{j+2}$, a constant factor being neglected on the right.

In order that $f_{p m}$ should be a perfect $m$ th power, $x_{2}^{m} \phi_{0 x_{1} \mid x_{2}}$ and the $p-2$ binary forms $X^{(j)}(j=1,2, \cdots, p-2)$ must be $m$ th powers. The vanishing of $H \phi_{0}$ and the $p-2$ Hessian covariants of $X^{(j)}(j=1,2, \cdots, p-2)$ are necessary and sufficient conditions for this.

The Hessian of $X^{(j)}$ is

and we have

$$
K_{j}=\left|\begin{array}{ll}
X_{x x}^{(j)} & X_{x y}^{(j)} \\
X_{y x}^{(j)} & X_{y y}^{(j)}
\end{array}\right|,
$$

$$
\begin{aligned}
& X_{x x}^{(j)}=m \sum_{h=0}^{m-2}(m-h)(m-h-1) a_{m-h 0 \ldots 0}^{\stackrel{j}{j} \overbrace{h-\ldots 0}^{p-j-2}} x^{m-h-2} y^{h},
\end{aligned}
$$

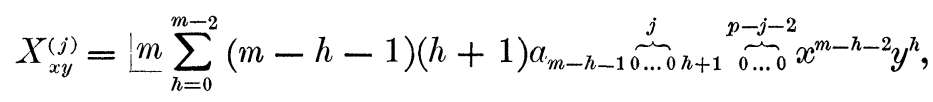

$$
\begin{aligned}
& X_{y y}^{(j)}=\left\lfloor m \sum_{h=0}^{m-2}(h+1)(h+2) a_{m-h-2} \underset{0_{0 \ldots 0 h+2}}{j} \underset{0 \ldots 0}{p-j-2} x^{m-h-2} y^{h},\right.
\end{aligned}
$$

formulas which give explicitly, in terms of the coefficients of $f_{p m}$, the values of the determinants $K_{j}$.

Since the $p-1$ binary forms $x_{2}^{m} \phi_{0 x_{1} \mid x_{2}}, X^{(j)}(j=1,2, \ldots$, $p-2)$ are all independent, the $p-1$ Hessians are all independent. Thus a set of necessary and sufficient conditions that a p-ary form $f_{p m}$ which is linearly factorable, should be the mth power of a linear form consist in the simultaneous vanishing of the $p-1$ binary Hessian covariants $H \phi_{0}, K_{j}(j=1,2, \cdots, p-2)$.

It will be proved in $\S 4$ that when the $\left(\begin{array}{c}m+p-1 \\ p-1\end{array}\right)-m(p-1)-1$ conditions that the general $p$-ary mic be linearly factorable are joined to the above $p-1$ Hessians we get a minimum set of conditions that $f_{p m}$ (in general) be an $m$ th power.

\section{§ 3. Conditions that $f_{3 m}$ Contain a Squared Factor.}

If we put $y=1, m=3, \alpha_{1}=\alpha_{2}=\alpha_{3}=1$ in (2), carry out the indicated multiplications and divide by the coefficient of $x^{3}$, we get 


$$
\begin{aligned}
f_{33} & \equiv \prod_{i=1}^{3}\left[x_{1}+r_{i} x_{2}-\frac{\phi_{1-r_{i}}}{\phi_{0-r_{i}}^{\prime}}\right]+\left(a_{10}-\sum \frac{\phi_{1-r_{1}} \phi_{1-r_{2}}}{\phi_{0-r_{1}}^{\prime} \phi_{0-r_{2}}^{\prime}}\right) x_{1} \\
& +\left(a_{01}-\sum \frac{\phi_{1-r_{1}} \phi_{1-r_{2}}}{\phi_{0-r_{1}}^{\prime} \phi_{0-r_{2}}^{\prime}} r_{3}\right) x_{2}+\left(a_{00}+\frac{\phi_{1-r_{1}} \phi_{1-r_{2}} \phi_{1-r_{3}}}{\phi_{0-r_{1}}^{\prime} \phi_{0-r_{2}}^{\prime} \phi_{0-r_{3}}^{\prime}}\right)
\end{aligned}
$$

where the summations are to be taken with reference to the $r_{i}$, being symmetric in the $r_{i}$. Since $f_{33}$ is factorable by hypothesis, the quantities in the parentheses are equal to zero. In fact these expressions equated to zero furnish a set of necessary and sufficient conditions that the general $f_{33}$ be factorable into linear factors distinct term for term.

If we write

$$
\begin{aligned}
& \Delta_{1}=3 a_{30} \frac{\partial}{\partial a_{20}}+2 a_{21} \frac{\partial}{\partial a_{11}}+a_{12} \frac{\partial}{\partial a_{02}}, \\
& \Delta_{2}=3 a_{03} \frac{\partial}{\partial a_{02}}+2 a_{12} \frac{\partial}{\partial a_{11}}+a_{21} \frac{\partial}{\partial a_{20}},
\end{aligned}
$$

and let $D$ be the discriminant of $x_{2}^{3} \phi_{0} x_{1} / x_{2}$ and $R_{3}$ the resultant of the latter taken with $x_{2}^{2} \phi_{1 x_{1} \mid x_{2}}$, these three conditional relations become

$$
\begin{aligned}
& H_{3}=a_{00} D-R_{3}=0, \\
& H_{2}=a_{01} D-\Delta_{2} R_{3}=0, \\
& H_{1}=a_{10} D+\Delta_{1} R_{3}=0 .
\end{aligned}
$$

If a ternary form $f_{33}$ contains a squared factor it is, of course, linearly factorable as a whole. Then (2) gives

$$
\begin{aligned}
f_{33}= & {\left[x_{1}+r_{1} x_{2}-\frac{\phi_{1-r_{1}}^{\prime}}{\phi_{0-r_{1}}^{\prime \prime}}\right]^{2}\left[x_{1}+r_{2} x_{2}-\frac{\phi_{1-r_{2}}}{\phi_{0-r_{2}}^{\prime}}\right] } \\
& +\left(a_{10}-2 \frac{\phi_{1-r_{1}}^{\prime} \phi_{1-r_{2}}}{\phi_{0-r_{1}}^{\prime \prime} \phi_{0-r_{2}}^{\prime}}-\frac{\phi_{1-r_{1}}^{\prime 2}}{\phi_{0-r_{1}}^{\prime 2}}\right) x_{1} \\
& +\left(a_{01}-2 \frac{\phi_{1-r_{1}}^{\prime} \phi_{1-r_{2}}}{\phi_{0-r_{1}}^{\prime \prime} \phi_{0-r_{2}}^{\prime}} r_{1}-\frac{\phi_{1-r_{1}}^{\prime 2}}{\phi_{0-r_{1}}^{\prime 2}} r_{2}\right) x_{2}+\left(a_{00}-\frac{\phi_{1-r_{1}}^{\prime 2} \phi_{1-r_{2}}}{\phi_{0-r_{1}}^{\prime \prime 2} \phi_{0-r_{2}}^{\prime}}\right) .
\end{aligned}
$$

Evaluating the expressions in the parentheses we get a minimum set of conditions that the general $f_{33}$ contain a squared factor, e. g., 


$$
\begin{aligned}
D & =0, \quad R_{3}=0, \\
\Xi_{1} & =a_{10} \frac{\partial D}{\partial a_{3-i i}}+\Delta_{1} \frac{\partial R_{3}}{\partial a_{3-i i}}=0, \\
\Xi_{2} & =a_{01} \frac{\partial D}{\partial a_{3-i i}}-\Delta_{2} \frac{\partial R_{3}}{\partial a_{3-i i}}=0, \\
\Xi_{3} & =a_{00} \frac{\partial D}{\partial a_{3-i i}}-\frac{\partial R_{3}}{\partial a_{3-i i}}=0, \\
& (i=\text { any number of the set } 0,1,2,3) .
\end{aligned}
$$

In $\S 2$ we have already developed a set of conditions that $f_{33}$ be a perfect cube, assuming it to be linearly factorable. The method of this section gives us a minimum set, without assumption. For if the resultant and the first subresultant of the first partial derivatives of $x_{2}^{3} \phi_{0 x_{1} / x_{2}}$ vanish, say $D=0, D_{1}=0$, and also the resultant and the first subresultant of $x_{2}^{3} \phi_{0 x_{1} / x_{2}}$ and $x_{2}^{2} \phi_{1 x_{1} \mid x_{2}}$, say $R_{3}=0, S_{3}=0$, then (2) gives

$$
\begin{aligned}
f_{33}=\left[x_{1}+r_{1} x_{2}-\frac{\phi_{1-r_{1}}^{\prime \prime}}{\phi_{0-r_{1}}^{\prime \prime \prime}}\right]^{3} & +\left(a_{10}-3 \frac{\phi_{1-r_{1}}^{\prime \prime 2}}{\phi_{0-r_{1}}^{\prime \prime \prime 2}}\right) x_{1} \\
& +\left(a_{01}-3 r_{1} \frac{\phi_{1-r_{1}}^{\prime \prime 2}}{\phi_{0-r_{1}}^{\prime \prime \prime 2}}\right) x_{2}+\left(a_{00}+\frac{\phi_{1-r_{1}}^{\prime \prime 3}}{\phi_{0-r_{1}}^{\prime \prime \prime 3}}\right),
\end{aligned}
$$

from which we get the necessary and sufficient conditions that the general ternary cubic be a cube, in the form

$$
\begin{aligned}
D & =0, \quad D_{1}=0, \quad R_{3}=0, \quad S_{3}=0, \\
\Gamma_{1} & =a_{10} \frac{\partial^{2} D}{\partial a_{3-i i}^{2}}+\Delta_{1} \frac{\partial^{2} R_{3}}{\partial a_{3-i i}^{2}}=0, \\
\Gamma_{2} & =a_{01} \frac{\partial^{2} D}{\partial a_{3-i i}^{2}}-\Delta_{2} \frac{\partial^{2} R_{3}}{\partial a_{3-i i}^{2}}=0, \\
\Gamma_{3} & =a_{00} \frac{\partial^{2} D}{\partial a_{3-i i}^{2}}-\frac{\partial^{2} R_{3}}{\partial a_{3-i i}^{2}}=0 \\
& (i=\text { any number of the set } 0,1,2,3) .
\end{aligned}
$$

We will now show still another method of applying the 
theorem of $\S 2$ in the determination of conditions for multiple factors of a ternary form $f_{3 m}$.

Consider the case where $f_{3 m}$ has a single linear factor of multiplicity 2 and let it have, besides, $m-2$ distinct linear factors. Then in (2) $\alpha_{1}=2, \alpha_{2}=\alpha_{3}=\cdots=1$, and

$$
\nabla_{r_{1}}=\phi_{1-r_{1}}^{\prime 2}-2 \phi_{0-r_{1}}^{\prime \prime} \phi_{2-r_{1}}=0 \text {. }
$$

Hence $f_{3 m}$ will have squared factors (and none of multiplicity higher than 2) when and only when there exist roots $r_{i}$ common to the three equations

$$
\phi_{0-r}=0, \quad \phi_{0-r}^{\prime}=0, \quad \nabla_{r}=0,
$$

provided the resultant $R$ of $\phi_{0-r}$ and $\phi_{0-r}^{\prime \prime}$ does not vanish. Thus if $D$ is the discriminant of $\phi_{0-r}$, and $\bar{R}_{1}$ is the resultant of $\nabla_{r}$ and the greatest common divisor of $\phi_{0-r}$ and $\phi_{0-r}^{\prime}$, a set of necessary and sufficient conditions that a linearly factorable $f_{3 m}$ should contain linear factors to the second power is

$$
D=0, \quad R \neq 0, \quad R_{1}=0 .
$$

\section{§ 4. Proof that the Conditions Give Minimum Sets.}

We can prove that the $\left(\begin{array}{c}m+p-1 \\ p-1\end{array}\right)-m(p-1)-1$ factorability conditions for any general $f_{p m}$ together with the $p-1$ Hessian conditions $H \phi_{0}=0, K_{j}=0(j=1,2, \cdots, p-2)$ of $\S 2$ furnish a minimum set of invariant conditions in order that the general $f_{p m}$ be an $m$ th power.

The number of identical relations which exist among the elementary symmetric functions of $m$ groups of $p$ homogeneous variables is $\left(\begin{array}{c}m+p-1 \\ m\end{array}\right)-1$. If these variables are the $m p$ coefficients of $m$ linear factors of $f_{p m}$, the elementary symmetric functions are the coefficients of that form. If the factors are distinct, term for term, the identical relations, considered as relations among the coefficients of the form, will be $\left({ }^{m+p-1}\right)-1$ equations in $m(p-1)$ non-homogeneous variables, so that only

$$
\left(\begin{array}{c}
m+p-1 \\
m
\end{array}\right)-m(p-1)-1
$$

of the relations are independent. An independent set forms a minimum set of conditions that $f_{p m}$ be factorable into $m$ linear factors, distinct term for term. ${ }^{p}$ Now if $f_{p m}$ is an $m$ th power, the number of variables is only $p-1$. Hence the minimum 
number of conditions in order that $f_{p m}$ may be an $m$ th power is $(\stackrel{m+p-1}{m})-p$.

Each one of the Hessians $H \phi_{0}, K_{j}(j=1,2, \cdots, p-2)$ is of order $2 m-4$ in the variables which it contains, and so the number of vanishing coefficients in each is $2 m-3$. Hence these give $(2 m-3)(p-1)$ conditions in addition to the $\left(\begin{array}{c}m+p-1 \\ m\end{array}\right)-m(p-1)-1$ assumed ones. But of the $2 m-3$ conditions obtained by equating to zero the coefficients of a binary Hessian covariant only $m-1$ are independent, as the $m$ coefficients of the form can all be expressed in terms of a single quantity when the Hessian vanishes. Hence we have as a total number of conditions given by the original factorability conditions of $f_{p m}$ and the Hessians

$$
\left(\begin{array}{c}
m+p-1 \\
m
\end{array}\right)-m(p-1)-1+(m-1)(p-1)=\left(\begin{array}{c}
m+p-1 \\
m
\end{array}\right)-p,
$$

which is thus the minimum number required. Hence the relations derived in $\S 2$ furnish a minimum set.

In the same way it may be shown that $(6),(7),(8),(9)$ are all minimum sets.

The University of Pennsyluania, Philadelphia, Pa.

\section{THE GENERAL TERM OF A RECURRING SERIES.}

BY PROFESSOR ARTHUR RANUM.

(Read before the San Francisco Section of the American Mathematical Society, September 26, 1908.)

1. The principal theorem of this note expresses the general term of a recurring series rationally in terms of the first few terms and the constants of the scale of relation. Although I derived it in 1908, I have only recently learned that practically the same theorem was published by D'Ocagne in 1894 (Journal de L'Ecole Polytechnique, volume 64, pages 151-224) and by Netto in 1895 (Monatshefte für Mathematik und Physik, volume 6, pages 285-290). Nevertheless it may be worth while to publish my own work for three reasons: first, because my proof is simpler than those of D'Ocagne and Netto ; second, because I have stated the result in a more explicit form than that of either of these authors*; third, because I have applied

* D'Ocagne gives an explicit statement of the theorem (p. 163) for the special case in which the series is a "suite fondamentale," but not for the general case. 